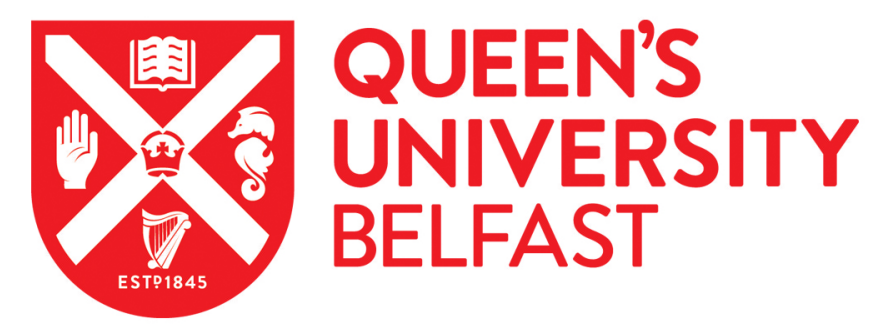

\title{
Current understanding of learning psychomotor skills and the impact on teaching laparoscopic surgical skills
}

White, C., Rodger, M., \& Tang, T. (2016). Current understanding of learning psychomotor skills and the impact on teaching laparoscopic surgical skills. The Obstetrician and Gynaecologist, 18(1), 53-63.

https://doi.org/10.1111/tog.12255

\section{Published in:}

The Obstetrician and Gynaecologist

Document Version:

Early version, also known as pre-print

Queen's University Belfast - Research Portal:

Link to publication record in Queen's University Belfast Research Portal

\section{Publisher rights}

(C) 2016 The Authors

This is the pre-peer reviewed version of the following article: White C, Rodger MWM, Tang T. Current understanding of learning

psychomotor skills and the impact on teaching laparoscopic surgical skills. The Obstetrician \& Gynaecologist 2016;18:53-63., which has been published in final form at [http://onlinelibrary.wiley.com/doi/10.1111/tog.12255/abstract. This article may be used for non-commercial purposes in accordance with Wiley Terms and Conditions for Self-Archiving.

\section{General rights}

Copyright for the publications made accessible via the Queen's University Belfast Research Portal is retained by the author(s) and / or other copyright owners and it is a condition of accessing these publications that users recognise and abide by the legal requirements associated with these rights.

\section{Take down policy}

The Research Portal is Queen's institutional repository that provides access to Queen's research output. Every effort has been made to ensure that content in the Research Portal does not infringe any person's rights, or applicable UK laws. If you discover content in the Research Portal that you believe breaches copyright or violates any law, please contact openaccess@qub.ac.uk. 


\section{Current understanding of learning psychomotor skills and the impact on teaching laparoscopic surgical skills}

Colette White MB BCh MRCOG, ${ }^{\mathrm{a}}$, ${ }^{*}$ Matthew WM Rodger PhD, ${ }^{\mathrm{b}}$ Thomas Tang MD MRCOGc aST7 Trainee in Obstetrics and Gynaecology, Royal Jubilee Maternity Services, Belfast Health and Social Care Trust, 274 Grosvenor Road, BelfastBT12 6BA, UK bLecturer in Psychology, Department of Psychology, Queen's University Belfast, Belfast BT9 5BN, UK

cConsultant Gynaecologist, Regional Fertility Centre, Royal Jubilee Maternity Services, Belfast Health and Social Care Trust, Belfast BT12 6BA, UK

*Correspondence: Colette White.

Email: colettewhite@doctors.org.uk

[Abstract]

\section{Key content}

- Trainees face many challenges in learning the skill set required to perform laparoscopic surgery.

- The time spent in the operating room has been detrimentally impacted upon since the implementation of the European Working Time Directive. In order to address the deficit, surgical educators have looked to the benefits enjoyed in the aviation and sports industries in using simulation training.

\section{Learning objectives}

- To summarise the current understanding of the neuropsychological basis of learning a psychomotor skill.

- To clarify factors that influence the acquisition of these skills.

- To summarise how this information can be used in teaching and assessment of laparoscopic skills.

\section{Ethical issues}

- The use of virtual reality simulators may be able to form a part of the aptitude assessment in the selection process, in order to identify trainees with the desired 
attributes to progress into the training programmes. However, as skill improves with practice, is it ethical to exclude novices with poor initial performance assessment before allowing them the opportunities to improve?

Keywords: laparoscopic surgery / psychomotor skills / simulator training / skill acquisition / surgery / training

\section{[Heading 1] Introduction}

Trainees face many challenges in their attempts to learn the skill set required to perform laparoscopic surgery. The learning curve is steep, the skills required are complex and trainees are faced with challenges of depth perception, visuospatial awareness, lack of direct feedback from tissuesand the fulcrum effect of the instruments. ${ }^{1,2}$ Teaching laparoscopic surgery is significantly more stressful for the surgeon than open surgery, as there is limited direct control over the trainee's actions. ${ }^{3}$ The number of hours spent in the operating room has been detrimentally impacted on since the implementation of the European Working Time Directive, Modernising Medical Careers, the increasing number of trainees and the changing nature of gynaecological surgery itself. ${ }^{1}$

The benefits of the laparoscopic approach are well documented and most gynaecological procedurescan be approached via the minimal access route. ${ }^{4}$ It is an essential skill for today's trainees to attain competence in, as emphasised by the Royal College of Obstetricians and Gynaecologists' Working Party Report Tomorrow's Specialist. $^{5}$

In order to address the deficit, surgical educators have looked into the benefit achieved in the aviation, music and sports industries in using simulation training. ${ }^{6}$ Several associations have adopted a curriculum of laparoscopic training such as the Society of American Gastrointestinal and Endoscopic Surgeons. It has established the Fundamentals of Laparoscopic Surgery (FLS) Program involving didactic teaching and simulation training, culminating in practical and theoretical exams that must be passed before registration with the American Board of Surgery. 
Gynaecological Endoscopy has developed the Gynaecological Endoscopic Surgical Education and Assessment diploma programme. This is based on five pillars of competence and three levels of expertise. It incorporates the theoretical and practical skills required of an endoscopic surgeon. The gateway to this is the web-based free tutorial programme called the Winner's Project. ${ }^{8}$ Nevertheless, completion of a compulsory simulation curriculum prior to and complementing live operating experience has not been universally implemented. ${ }^{2}$ This may be because of a lack of evidence that simulation improves training,patient safety and reduces healthcare costs. ${ }^{9}$ The Royal College of Obstetricians and Gynaecologists ${ }^{5}$ has acknowledged the important contribution of simulation training in both obstetrics and gynaecology. Simulation training has been endorsed as a method of counterbalancing the reduced working hours and operative experience of trainees.

Effective training strategy must incorporate robust quality assurances that retention of skills remains stable beyond the learning period. In order to progress from the safety of the laboratory to the operating room, skills learned must be transferrable to other potentially more complex situations, ${ }^{10}$ such as dealing with dense adhesions or haemorrhage. ${ }^{11}$

The purpose of this education article is to summarise the current understanding of the neuropsychological basis of learning a psychomotor skill, to clarify factors that influence the acquisition of these skills and to summarise how this information can be used in teaching and assessing laparoscopic skills.

\section{[Heading 1]Learning psychomotor skills}

\section{[Heading 2] Defining skill}

For present purposes, skill is defined as a stable and reliable link between perception of body and environment, and execution of goal-directed motor actions, which is both consistent across repeated performances of the action, and can be flexibly adapted to changes in task constraints. Skill in this sense is therefore considered primarily as a perceptual-motor function. Examples of perceptual-motor skills include instrumental 
music performance, driving a car, playing golf and so on. This category of skill might be contrasted with more cognitive or 'intellectual' skills, such as playing chess or mathematical reasoning. It is worth noting, however, that both categories of skill (perceptual-motor and intellectual) may share processes in common, ${ }^{12}$ and much may be learnt by considering the acquisition of expertise across a broad range of human achievement. Surgical performance requires capacities in common with other skilled behaviours, such as visual and haptic perception ('perception through touching'), finegrained physical and temporal movement, tool manipulation, and awareness of the overall task at hand through integrating these elements. For this reason and based on the definition given above, we will draw on a diverse range of activities to better understand the processes that underlie perceptual-motor skill learning.

\section{[Heading 2] Characteristics of skill acquisition}

Over the previous century, many psychological theories of skill acquisition have been proposed, such as those of Fitts and Posner, ${ }^{13}$ Anderson ${ }^{14}$ and Gentile. ${ }^{15}$ It is not germane within the scope of the present review to discuss each of these in detail here; rather we will try to identify commonalities across different theories, with the aim of highlighting psychological principles that are relevant to our domain of interest: laparoscopic surgery. In so doing, we will inevitably skim over subtleties and controversies that distinguish different psychological frameworks, but we hope that this approach will allow us to extract core concepts that may inform the development of effective training structures to support skill learning for surgeons.

Two common and related principles across different theories of skill acquisition are that:

1. Changes in performance due to training and practice proceed in anon-linear manner.

2. As the trainee advances in perceptual-motor learning, the way that they process the task and action will change through qualitatively different phases. 
The first principle is illustrated by the commonly observed learning curves, in which improvements in the performance outcomes (such as increased speed, reduced error) will be rapid during early trials, but then decelerate with increased practice. ${ }^{16}$ This slowing down of improvement rate as learning progresses may be due to a number of factors. First, in early stages, there is greater room for improvement, as the task is completely new, so there will be more likelihood of dramatic changes in performance. As the participant practises, the degree to which there is room for improvement will be less, and learning entails fine-tuning of the system to the task at hand. Second, and related to this, the processes underpinning skill acquisition appear to change as the novice moves through intermediate and advanced stages of learning. ${ }^{17,18}$ As neural control of skilled action moves from recruiting more conscious, executive resources (prefrontal cortex), to more automatic centres (basal ganglia), it can be expected that changes in learning speeds reflect differences in the processes of different neural structures. ${ }^{19}$ Hence, for a novice, skill learning involves large improvements as they introduce their nervous system to the new task arena, whereas skill acquisition for the more advanced learner involves slower refinements of already-learnt processes.

The second principle - that learners come to process the task in qualitatively different ways with advanced skill acquisition - is often characterised by a stage-based account of learning. For example, learners in an early stage of skill acquisition will rely on explicit rules about the action and the information relevant to the task, whereas more advanced learners will have developed implicit associations between task-relevant information and appropriate motor responses. ${ }^{13,20}$ For the novice, perception-action links are not automatic and 'hard-wired', therefore successful performance will be conscious, effortful, and require cognitive resources. With sustained, well-directed practice, such perception-action links should become more automatic, freeing up other mental resources to focus on future, external goals and adapt to perturbations in the environment. This final stage of skill acquisition is often termed 'automaticity', in which the component subskills of the task can be performed in an effortless, 'offline' manner. Automaticity in skill is characterised by less cognitive effort, less sensitivity to internal 
and external stressors, and of automatic skills being harder to modify once learnt. ${ }^{21}$ It is worth stating that the concept of automaticity does not mean that the skilled performer acts as an unthinking robot involuntarily following an over-rehearsed routine. Rather, the expert adapts the now easy-to-performsubunits of perception and action towards conscious goals and evolving task constraints. ${ }^{22}$

In perceptual-motor skill acquisition, many of the processes involved can be characterised as 'tuning' the goal-directed nervous system to the constraints of the task. This tuning process applies to movement, perception and attention, each of which is discussed in turn.

\section{[Heading 3] Movement}

A fundamental property of perceptual-motor learning is the improvement of precision and consistency of the spatial-temporal control of limbs. In learning to play a guitar, the practising musician must come to get their fingers to the right place, at the right time, and to pluck the string with the correct force. These factors can become highly precise and consistently repeatable over many instances - indeed, this is the impressive aspect of skill. However, even in highly practised movements, there is still variability in motor production. Consider an easy action like reaching out to pick up a cup: there are many ways that the different limb segments can extend and rotate to achieve this, and they will not be identical every time. ${ }^{23,24}$ The important outcome of learning is that variance at the end point of the action is minimal, regardless of variance elsewhere in moving limbs. This has traditionally been conceived as the 'motor redundancy' problem, ${ }^{23}$ although some researchers prefer to characterise this as 'abundancy'. ${ }^{25}$ Through practice, the learner's nervous system will tune into the space of outputs that maximise precision and invariance in the task-relevant dimensions. ${ }^{26}$ While

there is much debate about exactly how the brain achieves this, ${ }^{27-29}$ one thing that seems clear is that the motor system needs to explore the space of movement possibilities in order to zone in on those that are most aligned withtask success. ${ }^{26,30}$ This may account for the finding that greater motor variability early on in skill learning is 
associated with accelerated learning later on. ${ }^{31}$ Hence, allowing the trainee surgeon to fully explore the possible movement space early on may lead to improved motor control outcomes later.

Another characteristic of skill acquisition is that as a result of learning, the trainee's movements become qualitatively different compared with their earlier performance attempts. Movement quality is smoother and less jerky, ${ }^{32,33}$ limb segments are coordinated in a more efficient manner, ${ }^{34}$ and sequences of individual submovements become grouped together to form more complex wholes. ${ }^{35}$ These points all indicate that differences should be expected in the actual quality of movement produced by more skilled surgeons.

\section{[Heading 3] Perception}

As well as creating the correct movement output, essential to the achievement of skill is sensitivity to perceptual information about the changing position of the body in relation to the desired end point and also, task-relevant external factors. It can be seen that perception of the task is very different between novice and experts. Whereas novice chess players view the board in terms of the individual pieces and their current position, the expert player sees the board as a unified pattern, with particular opportunities for future moves. ${ }^{36}$ Moreover, such differences in perception are tuned into the task at hand. In football, more skilled goalkeepers visually sampled curved free kicks for longer than novices, with the result that their actions were not as susceptible to the deceptive motion in the early part of the ball trajectory. ${ }^{37}$ Experts are also more sensitive to the useful perceptual information available and can use this to anticipate future events. This may explain the benefits of 'implicit learning': in the absence of explicit rules, the trainee has to abstract from the patterns in their sense the variables that directly specify the appropriate (successful) action. 
Related to perception is the concept of attention in skill acquisition, that is, what information the learner focuses on. In sight-reading (playing music from a score that has never been seen before), more skilled piano players look further ahead, taking in a greater bandwidth of information and attending to future events. ${ }^{38}$ This tuning of attention gives the skilled performer an advantage in maximising the efficiency of cognitive resources, which is important as such resources may be reduced in stressful or unpredictable situations. ${ }^{39}$ It is also worth noting that expert performers generally have an external focus of attention, concentrating on the environment and the task goal, while novices tend to focus on their own movements. ${ }^{40}$ This is important, because it has been found that expert performance can be adversely affected by switching attention to internal processes, often disastrously so. ${ }^{41,42}$ In intermediate stages of learning, attention may switch between internal processes and external factors. ${ }^{43}$

\section{[Heading 2] Linking movement to what you see}

A very relevant area of research into perceptual-motor skill acquisition is the study of scenarios in which perception and action are decoupled from their natural order. Visuomotor adaptation occurs when vision of one's own body and the environment are dislocated, as occurs in laparoscopic surgery, when the surgeon has to adapt to the decoupling of their motor commands and vision of their actions. Studies demonstrate that after a small number of error trials, participants can adapt to these perceptual constraints. ${ }^{44}$ Also, on removal of the visual disruption, there is an adaptation aftereffect in which compensatory corrections cause the action to make errors in the opposite direction. The number of trials needed to 'wash-out' this effect depends on the degree of adaptation that occurred. It has been found that variable practice (switching the visual perturbation or target between trials) can lead to faster and more effective visuomotor adaptation than blocked practice (multiple repeated trials of each perturbation or target). Hence, in laparoscopic simulation practice, time should be given for this adaptation to occur.Increasing variability between practice trials may improve 
the speed with which adaptation occurs. For example, the set-up of the laparoscopic training box and the monitor should closely resemble 'real' theatre settings.

\section{[Heading 2] Importance of error and exploration}

In all the processes of skill acquisition discussed here, one common principle is the idea that the learner needs to be able to explore the possible space of actions and outcomes, making errors, in order to establish a reliable and adaptable action repertoire. Increased variability and error during practice allows the learner to experience a broader range of perceptual motor variables, making them more sensitive to those that align with successful action. Moreover, by training the learner to tune into the information that specifies the properties of the task, they will be better able to attend to these when performing in new settings, or when the environment is full of distracting task-irrelevant information, as may be the case in an operating theatre.

\section{[Heading 1] Factors influencing the acquisition of skills, and performance of laparoscopic surgery}

\section{[Heading 2] Practice and schedule}

It is intuitive that practice should improve technical skills, however, it is the quality, not quantity of practice that influences resilience. ${ }^{44}$ For trainees with limited free time, the way that they practise should be efficient and facilitate transference of the skills to reallife tasks. Once learnt, it is important that laparoscopic skills are maintained over time. Skills learnt through simulation in obstetrics and internal medicine are maintained several months following the teaching episode. ${ }^{45}$ However, there is evidence that laparoscopic skills can deteriorate over time if not practised. ${ }^{46}$

Practice that is repetitive, mindful of the task, focused on a well-defined learning objective is called deliberate practice and is important in the development of expert skill. ${ }^{45,47,48}$ The learner should constantly monitor their performance and correct their 
strategy accordingly. ${ }^{47}$ Varying the skill practised will promote stability in learning, ${ }^{47,49}$ enable the trainee to apply learnt skills to different scenarios and allow the trainee to become more able to adapt to different challenges that they may face in live operating. ${ }^{49}$ Varying the complexity of the skills learnt and increasing the level of difficulty can enhance learning on simulators. ${ }^{2,47,50}$ This strategy may also help to stimulate trainees' interest and therefore motivation. ${ }^{2}$ Skills practised should be individualised to the trainee's level of expertise..$^{45}$ In the early stages of learning when there is a high demand on cognitive resources, repeated failure of a task can heighten frustration and hinder acquisition of skills. ${ }^{51}$ Trainees should start with basic tasks initially and gradually move on to more complex procedures ${ }^{49,52,53}$ when they are more able to cope with the complexity. ${ }^{44}$

When learning to execute a motor skill, novices need to think about each step. ${ }^{47,54}$ They are less able to deal with other distractions and tire quickly. ${ }^{53}$ The aim of practising a procedure or task is to become so proficient in its execution that the steps required take little cognitive processing and are intuitive. ${ }^{47}$ Focusing on the outcome of the manoeuvre (the external focus) rather than the steps involved in carrying out the manoeuvre, results in more effective performance and learning. ${ }^{11,52,53}$ For example, when teaching how to tie a knot, traditionally the emphasis may have been on describing the movement of the suture thread and placement of the fingers. This results in an internal focus of attention and is ineffective. ${ }^{11}$ More effective instruction would be to describe the shape that the suture thread should create, in other words, creating ' $\mathrm{C}$ ' shapes with the suture material in the instruction of intracorporeal knot tying. ${ }^{55}$

Trainees have different learning curves for different tasks and the time and number of repetitions to achieve competency may vary. Rather than specify an amount of time in training, acquisition of the desired skill should determine proficiency. ${ }^{56}$ Distributed training, with rest between sessions, is superior to massed training for obtaining psychomotor skills, ${ }^{47,57}$ and for long-term retention of the skills. ${ }^{44,51}$ Training for more 
than 45 minutes in duration is likely to result in a decrease in concentration and accuracy and therefore should be avoided. ${ }^{51}$

\section{[Heading 2] Learning in pairs}

Neuroimaging has shown that the same neural structures are activated during action production and observation of the action. Learning in pairs, with alternation between observation and physical practice, enhances learning and leads to greater transferability. This may be as a consequence of direct competition between the pairs and setting of higher goals. ${ }^{11}$ It may prove to be a more enjoyable way to learn and is certainly more cost-effective.

\section{[Heading 2] Warm up}

Warming up at least 15 minutes before the surgical procedure decreases operating time and error. This beneficial effect should last for 30 minutes and is exhibited in novices and experts alike. During a surgical list, the warm-up process should be carried out before each case. ${ }^{58}$

The presence of simulators close to the operating theatre would allow the trainee to practise their skills opportunistically between cases and enhance their performance if they are given the opportunity to operate. This also allows them to be supervised by consultants who are likely to be motivated and knowledgeable and who can provide them with immediate feedback as to how their practice has impacted their surgical performance.

\section{[Heading 2] Supervision and feedback}


In order to promote effective learning there must be specific, challenging but achievable goals and outline steps required to reach that goal. ${ }^{44}$ Goals should be set that are relevant to the trainees' stage of learning and not be overwhelming in order to be motivational. ${ }^{44,48}$

Effective instruction will allow trainees to develop a mental model of how to approach the task and is crucial in motor skill learning. ${ }^{47}$ Video-based education has been shown to aid faster acquisition of skill on surgical simulators. ${ }^{47}$ This is maximised when video tutorials are used before and during training. ${ }^{47}$ However, supervision may prevent picking up bad habits and can aid in the acquisition of skills, in particular suturing. ${ }^{59}$

During the execution of a motor skill the learner receives visual, auditory and haptic feedback on their performance. Interventions that improve the internal feedback of the performer can aid acquisition of skill. ${ }^{47}$ For example, the incorporation of haptic feedback into virtual reality simulators improves task performance compared with training without haptic feedback ${ }^{47}$ High-fidelity virtual reality simulators can also provide feedback by other visual alarms, for example, the screen flashes a red colour when tissue trauma has occurred. ${ }^{60}$

Feedback given while the task is being carried out may be advantageous for novice learners at the start of their training, ${ }^{44}$ and directly improve clinical performance ${ }^{45}$ by immediately correcting misperceptions. ${ }^{44}$ However, this kind of feedback can distract trainees from the task at hand ${ }^{44}$ and may detrimentally affect task resilience. ${ }^{2}$ As learning progresses, immediate feedback should gradually be reduced in favour of feedback given at the end of the performance. ${ }^{44,47}$ During live operating direct supervision is essential, especially during the initial learning curve. Feedback at this stage is expected to be instant and frequent. As learning progresses and the trainee's skills improve, it may be appropriate to discuss the training opportunity after the theatre session to allow the trainee to concentrate on the task at hand, observing patient safety as a priority at all times. ${ }^{44}$ Feedback given less than $100 \%$ of the time is 
optimal but the ideal intensity and timing of feedback is not known. ${ }^{44}$ More frequent feedback appears to lead to faster acquisition of skills whereasfewer feedback episodes are associated with greater retention. ${ }^{44}$ Practice time without feedback will allow learners to develop their own learning strategies. ${ }^{11,44,47}$ Honest feedback that is positive and normative (comparing trainee to peers) is motivational. ${ }^{11}$ Trainees often have a feel for how they have performed, so concentrating on mistakes may be redundant and heighten concerns about themselves that may lowermorale and hamper learning. ${ }^{11}$

The interaction between trainee and trainer can affect the trainee's learning capabilities. A conflicted and mismatched relationship may hinder progression and negatively impact their enthusiasm and motivation to learn. ${ }^{48}$

\section{[Heading 2] Errors}

Knowledge is increased by making and learning from errors. When learning a motor skill, it is essential that there is opportunity to discover the relationship between the speed and accuracy of the movement required. Also, it is important to experience the specific movement organisation and execution strategies that lead to optimal outcomes.

Learning through error is not an acceptable learning philosophy to adapt in the operating theatre but is a circumstance when simulation practice comes into its own. During simulation, in order to assist the trainees in planning their movements, they can be allowed to create errors in order to become aware of their limitations.

\section{[Heading 2] Gaze training}

Research in sports skills learning has found that gaze training interventions may support the development of robust movement skills. ${ }^{61}$ Expert laparoscopic surgeons exhibit a characteristic gaze pattern when performing skills. They focus on the goal 
without the need to check their tool location. This is in contrast to novices whose gaze tends to switch between tracking their tool and fixating on the target. This is more cognitively demanding and leaves less ability to multitask. Research has demonstrated that using a video depicting an expert's gaze control during the completion of a task, it is possible to instruct trainees to carry out a task adopting this gaze pattern. This method of gaze training has been shown to enhance the speed by which a skill is obtained and the resilience of the skill to the demands of stressors during laparoscopic surgery and in sport. Gaze training also promotes the ability to transfer the skill to other more complex tasks and durability of the skill over time (Table 1). ${ }^{61-65}$

\section{[Heading 2] Handedness, gender and video gaming}

Handedness is important when discussing laparoscopic surgical skills as it influences dexterity and eye-hand coordination. ${ }^{66}$ There is a suggestion that right-handed males have more innate dexterity than left-handed males, who have more dexterity than righthanded females. ${ }^{66}$ However, proficiency with the non-dominant hand can be improved with repetition of tasks and procedures. ${ }^{66}$ Simulation training may accelerate this process and video gaming also helps to improve dexterity. ${ }^{67}$ Some studies have shown that females perform less well on visuomotor tasks and have a poorer surgical skills rating, however, others have found no gender difference in the performance of a laparoscopic skill, ${ }^{68}$ and that differences diminish with practice. ${ }^{66-69}$

There is much interest regarding experience of video gaming and its relation to laparoscopic surgical aptitude. A systematic review by $\mathrm{Ou}$ et $\mathrm{al}^{70}{ }^{70}$ concluded that trainees with prior or current video-gaming experience have superior laparoscopic simulator performance. This was measured in terms of time to completion, improved efficiency and fewer errors when compared with non-gaming counterparts. When playing video games the user has to learn to adapt for the dissociation of their motor commands and the vision of their actions. Eventually they will not have to look at the 
controller in their hands but watch the screen, with responses becoming automatic. This skill development may be beneficial when faced with the similar challenges of laparoscopic surgery.

\section{[Heading 2] Video instruction}

Video-based instruction that trainees can access easily and at their discretion may improve the trainees' feeling of being in control of how they practise. Videos are easily accessed via the British Society of Gynaecological Endoscopy and WeBSurg websites, among others including YouTube. There is access to a vast array of surgical procedures teaching both choreography and variations in surgical technique. Watching the videos to help solve a problem, clarify an action or answer a question may improve the trainees' feeling of independent learning and increase their motivation. ${ }^{11,71}$

\section{[Heading 2] Cadaver and animal model training}

Learning laparoscopic skills on cadavers is beneficial in that they provide a realistic learning model comparable with live surgery onwhich trainees can complete full procedures. ${ }^{72}$ However, significant drawbacks include expense, lack of specimens and cadaver laboratories, ${ }^{73}$ and moral and ethical objections.

Although there is no animal model that perfectly represents human anatomy, laparoscopic surgical techniques have been successfully demonstrated on ewes, and live and freshly killed pigs. ${ }^{74,75}$ These skills have been shown to subjectively and objectively improve performance that has transferred to theatre. ${ }^{76}$ Similar drawbacks apply that pertain to the use of cadaver models. 


\section{[Heading 2] Environment}

The environment in which teaching takes place can affect optimal performance. Noise has been shown to impair effective communication and can increase knowledge-based errors during complex task performance.$^{77}$ Some surgeons enjoy music in their operating room and for experts it may enhance their concentration and boost performance. ${ }^{78}$ However, it does not enhance trainee performance and may even be distracting for trainee surgeons performing a new task. ${ }^{79}$ This may be amplified if trainees feel that they have no control over the theatre environment, including the choice of music.

Surgeons face multiple occupational stresses: time pressures, concerns regarding patient welfare, administrative duties, responsibility to train others, maintaining their own skills and working with different colleagues as a consequence of shift-pattern working. Additionally, they are exposed to considerable challenges when performing surgery. Teaching laparoscopic skills can be stressful for the surgeon because they have no control over the actions of the trainee; they can guide them verbally but unlike in open procedures they have no ability to direct movements physically. For novices their inexperience and lack of familiarity and control over their environment can be stressful. Some amount of stress can be beneficial and enhance alertness and task efficiency ${ }^{80}$ However, increasing stress can lead to increased number of errors, longer task completion time and greater number of unnecessary movements. ${ }^{81}$

\section{[Heading 2] Distractions}

When executing a motor skill, the appearance of a distractor in the visual field evokes the same temporal response as though moving toward the distractor. This can cause a delayed initiation of the intended action, or worse, with respect to surgery, movement toward the distractor. Every attempt should be made to remove distractors that have 
the potential to engage these competing response tendencies. ${ }^{49}$ For example, normalising anatomy by dividing adhesions prior to commencing the planned procedure will help reduce the potential for distractions.

\section{[Heading 2] Non-technical skills}

Simulation training that is fully integrated into an established educational programme will ensure that all the skills that contribute to competent surgical practice are developed. ${ }^{45} \mathrm{~A}$ competent surgeon must have clinical experience and knowledge of procedures, anatomy and possible complications. Good communication skills are paramount, as is the ability to work in a team because the role of the primary surgeon in an operating theatre is akin to the conductor of an orchestra.

It is essential that trainees are aware of the safe set-up of theatre and equipment. These skills are expected for live operating but it has also been demonstrated that errors made during simulation are often also a consequence of a knowledge gap, for example of the correct steps of a procedure. ${ }^{82}$ Trainees who learn practical surgical skills on a simulator alongside cognitive skills (anatomy, steps of procedure, errors and complications) outperform those who learn on a simulator alone.,.$^{2,83}$

\section{[Heading 1] Simulation training and assessment}

Development of a curriculum for laparoscopic simulation training must consider the characteristics of the learner. The potential for learning will be strengthened if trainees are given the autonomy to decide what and when they practise. However, they should have defined goals and learning objectives relevant to their stage of training and, in parallel, a current training syllabus. ${ }^{47}$ Surgical trainees are presumed to be motivated to improve their skills, by the fact that they have selected the specialty. However, in order 
to optimise their performance, attention should be paid to individual personality, confidence level and experience. Mandatory participation in simulation training may be the most effective motivator for trainees who have many other demands on their time ${ }^{47}$ because uptake of voluntary skills training is poor. ${ }^{47}$ Trainers should be motivated, have training in the area of simulation and be familiar with the simulator model being used.

Laparoscopic simulation can be performed using simple box trainers (camera to display the inside of the box and contents) or virtual reality simulators (computer simulation). Practice on a box trainer will improve technical skill; however, the use of virtual reality simulators reduces operation time and improves performance when compared with no training or use of a box simulator. ${ }^{9}$ The characteristics of these systems are outlined in Box 1.9,84,85

\section{[Heading 2] Summative assessment}

The benefits of different forms of feedback at different stages of the learning curve have been discussed. However, in order to prove that certain proficiencies have been met, trainees will require some method of objective summative assessment of their skills. This may be deemed necessary to progress to the next stage of their curriculum or to allow operating privileges.

Objective structured assessment of technical skills (OSATS) has been employed to evaluate surgical performance objectively for many years. However, the format is mainly considered to be valid as a measure of progress of training rather than examination or credentialising ${ }^{86}$ and has not been evaluated for assessment of simulation training. ${ }^{87}$ Expert review of unedited videos of simulation by two experts $^{88}$ combined with OSATS or similar criteria has been found to be a reliable ${ }^{89}$ and valid method of assessment of proficiency. ${ }^{90}$ 
Well-established training curricula, such as the FLS and Gynaecological Endoscopic Surgical Education and Assessment programmes, carry out assessments on adapted box simulators. The FLS trainer uses the MISTELS programme and theGynaecological Endoscopic Surgical Education and Assessment programme the LASTT system. ${ }^{88,89}$ These programmes have demonstrated feasibility, construct validity (ability to distinguish novice from expert) and transfer of skill to the operating room. ${ }^{88,89}$ The skills have also been shown to be resistant to decay and represent a good example of an evidence-based curriculum designed to teach basic laparoscopic skills. ${ }^{2}$

Use of a virtual reality simulator such as the LAP Mentor ${ }^{\mathrm{TM}}$ virtual reality laparoscopic simulator (Simbionix, Cleveland, OH, USA) may be used to provide summative assessment for novices and ultimately certification and credentialisation for expert surgeons. Construct validity for the LAP Mentor has been demonstrated for basic skills when evaluating the performance of medical students, residents and surgeons with different experience levels. ${ }^{90-95}$ It has also been able to identify the special skills, and identify areas of weakness, of expert surgeons, such as bariatric surgeons completing the gastric bypass module on the virtual reality simulator. ${ }^{90}$

\section{[Heading 1] Further research and ethical issues}

Several studies have confirmed the benefit of simulation training on improved laparoscopic surgical skills, in particular, accelerating the attainment of these skills and shortening the learning curve. ${ }^{4,96,97}$ Studies have concluded that skills learnt in

simulation training can be transferred to the operating theatre., $, 56,98-101$ However, this has not been shown to translate into better patient outcome or decreased healthcare costs. ${ }^{49}$ This relationship has been demonstrated in other areas of medical simulation. In obstetrics, the introduction of team-based simulation training resulted in improved neonatal outcome. ${ }^{102}$ Trainees who mastered the insertion of a central venous catheter 


\section{in a simulation laboratory experienced significantly fewer procedural complications in}

an intensive care unit than those who were not simulator trained.. Med Educ 2009;43:507-15.

72. Sharma M, Macafee D, Horgan AF. Basic laparoscopic skills training using fresh frozen cadaver: a randomized controlled trial. Am J Surg 2013;206:23-31.

73. Levine RL, Kives S, Cathey G, Blinchevsky A, Acland R, Thompson C, et al. The use of lightly embalmed (fresh tissue) cadavers for resident laparoscopic training. J Minim Invasive Gynecol 2006;13:451-6.

74. Lin E, Szomstein S, Addasi T, Galati-Burke L, Turner JW, Tiszenkel HI. Model for teaching laparoscopic colectomy to surgical residents. Am J Surg 2003;186:45-8.

75. Woods JR Jr, Ansbacher R, Castro RJ, Marshall W, Trabal JF. Animal surgery: an adjunct to training in obstetrics and gynecology. Obstet Gynecol 1980;56:373-6.

76. Scheeres DE, Mellinger JD, Brasser BA, Davis AT. Animate advanced laparoscopic courses improve resident operative performance. Am J Surg 2004;188:157-60.

777 Moorthy K, Munz Y, Dosis A, Bann S, Darzi A. The effect of stress-inducing conditions on the performance of a laparoscopic task. Surg Endosc 2003;17:1481-4.

78. Allen K, Blascovich J. Effects of music on cardiovascular reactivity among surgeons. JAMA 1994;272:882-4.

79. Miskovic D, Rosenthal R, Zingg U, Oertli D, Metzger U, Jancke L. Randomized controlled trial investigating the effect of music on the virtual reality laparoscopic learning performance of novice surgeons. Surg Endosc 2008;22:2416-20.

80. Klein G. Sources of Power. How People Make Decisions. Cambridge, MA: MIT Press, 1999.

81. Arora S, Sevdalis N, Aggarwal R, Sirimanna P, Darzi A, Kneebone R. Stress impairs psychomotor performance in novice laparoscopic surgeons. Surg Endosc 2010;24:2588-93.

82. Tang B, Hanna GB, Cuschieri A. Analysis of errors enacted by surgical trainees during skills training courses. Surgery 2005;138:14-20.

83. Van Herzeele I, Aggarwal R, Neequaye S, Darzi A, Vermassen F, Cheshire NJ. Cognitive training improves clinically relevant outcomes during simulated endovascular procedures. J Vasc Surg 2008;48:1223-30.

84. Barsuk JH, McGaghie WC, Cohen ER, O'Leary KJ, Wayne DB. Simulation-based mastery learning reduces complications during central venous catheter insertion in a medical intensive care unit. Crit Care Med 2009;37:2697-701.

85. Botden SM, Torab F, Buzink SN, Jakimowicz JJ. The importance of haptic feedback in laparoscopic suturingtraining and the additive value of virtual reality simulation. Surg Endosc 2008;22:1214-22.

86. van Hove PD, Tuijthof GJ, Verdaasdonk EG, Stassen LP, Dankelman J. Objective assessment of technical surgical skills. Br J Surg 2010;97:972-87.

87. Larsen C, Oestergaard J, Ottesen B, Soerensen J. The efficacy of a virtual reality simulation training in laparoscopy: a systematic review of randomized trials. Acta Obstetrica Gynecol Scand 2012;91:1015-28.

88. Campo CR, De Win G, Ritter O, Keckstein J, Miserez M, Campo R. Feasibility and construct validity of a novel laparoscopic skills testing and training model. Gynecol Surg 2008;5:281-90.

89. Campo R, Reising C, Van Belle Y, Nassif J, O'Donovan P, Molinas CR. A valid model for testing and training laparoscopic psychomotor skills. Gynecol Surg 2010;2:133-41.

90. Giannotti D, Patrizi G, Casella G, Di Rocco G, Marchetti M, Frezzotti F, et al. Can virtual reality simulators be a certification tool for bariatric surgeons? Surg Endosc 2014;28:242-8.

91. Iwata N, Fujiwara M, Kodera Y, Tanaka C, Ohashi N, Nakayama G, et al. Construct validity of the LapVR virtual-reality surgical simulator. Surg Endosc 2011;25:423-8.

92. McDougall EM, Corica FA, Boker JR, Sala LG, Stoliar G, Borin JF, et al. Construct validity testing of a laparoscopic surgical simulator. J Am Coll Surg 2006;202:779-87.

93. Schijven M, Jakimowicz J. Construct validity: experts and novices performing on the Xitact LS500 laparoscopy simulator. Surg Endosc 2003;17:803-10.

94. Yamaguchi S, Konishi K, Yasunaga T, Yoshida D, Kinjo N, Kobayashi K, et al. Construct validity for eye-hand coordination skill on a virtual reality laparoscopic surgical simulator. Surg Endosc 2007;21:2253-7. 
95. Zhang A, Hunerbein M, Dai Y, Schlag PM, Beller S. Construct validity testing of a laparoscopic surgery simulator (Lap Mentor): evaluation of surgical skill with a virtual laparoscopic training simulator. Surg Endosc 2008;22:1440-4.

96. Grantcharov TP, Kristiansen VB, Bendix J, Bardram L, Rosenberg J, Funch-Jensen P. Randomized clinical trial of virtual reality simulation for laparoscopic skills training. Br J Surg 2004;91:146-50.

97. Madan AK, Frantzides CT. Prospective randomized controlled trial of laparoscopic trainers for basic laparoscopic skills acquisition. Surg Endosc 2007;21:209-13.

98. Seymour NE, Gallagher AG, Roman SA, O'Brien MK, Bansal VK, Andersen DK, et al. Virtual reality training improves operating room performance: results of a randomized, double-blinded study. Ann Surg 2002;236:458-63.

99. Kundhal PS, Grantcharov TP. Psychomotor performance measured in a virtual environment correlates with technical skills in the operating room. Surg Endosc2009;23:645-9.

100. Dawe SR, Windsor JA, Broeders JA, Cregan PC, Hewett PJ, Maddern GJ. A systematic review of surgical skills transfer after simulation-based training: laparoscopic cholecystectomy and endoscopy. Ann Surg 2014;259:236-48.

101. McClusky DA, Gallagher AG, Ritter EM, Lederman AB, Van Sickle KR, Baghai M, et al. Virtual reality training improves junior residents' operating room performance: results of a prospective, randomised, double-blinded study of the complete laparoscopic cholecystectomy. J Am Coll Surg 2004;199:S73.

102. Draycott TJ, Crofts JF, Ash JP, Wilson LV, Yard E, Sibanda T, et al. Improving neonatal outcome through practical shoulder dystocia training. Obstet Gynecol 2008;112:14-20.

103. Buckley CE, Kavanagh DO, Gallagher TK, Conroy RM, Traynor OJ, Neary PC. Does aptitude influence the rate at which proficiency is achieved for laparoscopic appendectomy? J Am Coll Surg 2013;217:1020-7.

104. Thijssen AS, Schijven MP. Contemporary virtual reality laparoscopy simulators: quicksand or solid grounds for assessing surgical trainees? Am J Surg 2010;199:529-41. 
Box 1. Characteristics of virtual reality simulators and box trainers

\section{Box trainers}

Inexpensive

Some boxes have fixed camera/some mobile

Facilitates learning on inanimate objects/animal parts

Better realism - using actual laparoscopic instruments 9

Haptic feedback

Use of videos can complement training

Trainer required for supervision and feedback

Can be videoed for assessment/feedback

More amenable to suture practice 85

Virtual reality simulators

Expensive

No need for other equipment, eg sutures9,84

Able to practise complete surgical procedures

Some models incorporate haptic feedback

No requirement for second assistant

Immediate feedback available

Scores stored for monitoring of progress

Able to compare scores with peers

Can be unsupervised

Associated with a more enjoyable experience84

[end] 
[box]

Box 2. Strategies to optimise simulation training

- Easily accessible simulator in a quiet area free from interruptions

- Mandatory, frequent training sessions lasting less than 45 minutes

- Have access to additional learning aids, eg videos of procedure

- Learn in pairs

- Have access to motivated tutor

- Initial introduction to simulator:

o How it works

o Overview of tasks amenable to type of simulator

- Practise tasks appropriate to ability:

o Start with simple tasks

o Progress to complete procedures

- Vary skills practised

- Set goals of skills to be learnt

- Opportunity for feedback

- Concurrent teaching on anatomy, procedures, complications, etc.

- Continued practice to maintain skills

[end]

Table 1. Evidence for gaze training

\begin{tabular}{lll}
\hline Study & Participants & Methods \\
\hline Wilson et al. & 30 medical students & Baseline attempt at the hand-eye coordination task on \\
$(2011)^{61}$ & with no laparoscopic & LAPMentor before randomisation to: \\
& experience & 1. Gaze trained: watched a video with expert's gaze depicted \\
& with cursor and given feedback after every attempt $(n=10)$ \\
& 2. Movement trained: watched same video with cursor \\
& removed but movements verbally described, given feedback \\
& after every attempt $(n=10)$ \\
& 3. Discovery learning (control group): given no video, verbal \\
& instruction or feedback $(n=10)$ \\
& After training a control task assessed learning, a
\end{tabular}

Results

Gaze-trained group learned more

quickly, with performance difference more pronounced while performing concurrent cognitive task $(P<0.001)$ 
Vine et al. (2012) $22 \quad 27$ novices with no laparoscopic experience

Vine et al. (2013)63

Vine et al. $(2010)^{64}$

Vine et al. (2011) ${ }^{65}$

16 basketballnovices

Vine et al. (2011) $65 \quad 22$ elite golfers

14 inexperienced golfers multitasking transfer test assessed resilience

Randomised to two groups:

1. Gaze trained used surgery-training template placed over monitor to guide expert-like gaze by highlighting key locations on screen $(n=14)$

2. Discovery learning group had a normal view of the monitor $(n=13)$

Neither group was given instruction. Each group completed 50 trials of ball pick up and drop test on 3Dsim minimally invasive training system. A retention task was carried out then the task was completed under stress; time pressure, competitive and evaluation-threat conditions

Randomised to gaze trained (GT) $(n=18)$ or discovery

learning (DL) $(n=18)$ groups. 50 trials of picking up and dropping balls one handed, then two-handed grasping and cutting task. Participants performed baseline, retention and delayed retention (one month) tests. Completion time and gaze control were assessed

Randomised to gaze trained (GT) $(n=7)$ or control group $(n=7)$. Both groups given technical advice on how to putt golf ball but GT group also given detailed instruction regarding where to direct gaze. Each group carried out 40 pre-test putts, a series of 320 acquisition phase putts, 40 retention, 40 transfer (under conditions creating anxiety) and then 40 retention putts

40 pre-test throws then randomised to gaze trained $(\mathrm{GT})(n=8)$ and control groups $(n=8)$. Both groups were given technical instruction, GT group instructed specifically on gaze strategy. Then each carried out 360 throws during training period followed by 120 throws under stressful conditions

Gathered putting statistics over 10 rounds of competitive golf and were then randomised to gaze trained (GT) $(n=11)$ or control group $(n=11)$. Then 20 pre-test putts, followed by 20 training putts. Videofeedback after each putt was given to each group but GT group were given additional instruction related to maintaining an optimal gaze. Putting statistics were then recorded over 10 competitive rounds. Further lab tests of retention and pressure performance
Surgical training template was successful in guiding gaze-trained group to adopt expert-like gaze strategy which improved performance with better completion time and fewer errors in both retention and stress tests

The gaze-trained group consistently displayed a more expert-like gaze control and superior performance in retention task $(P \leq 0.05)$ and in delayed retention task $(P \leq 0.001)$

GT group performed better under pressure and had more attentional control

GT group performed significantly better in pressure test comparedwith control group $(P \leq 0.001)$

GT group maintained skills under pressure $(\mathrm{P} \leq 0.005)$ control group made no change to putting stats after training 